\title{
8 Buying Time: Plasticity and Population Persistence
}

\author{
Sarah E. Diamond and Ryan A. Martin \\ Case Western Reserve University
}

\section{CONTENTS}

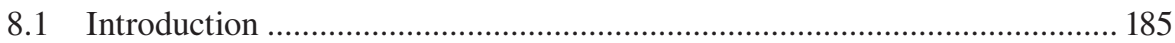

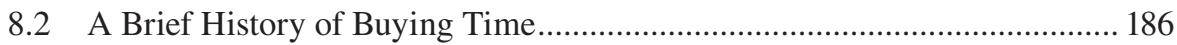

8.3 The Theory and Modeling Behind Buying Time ......................................... 187

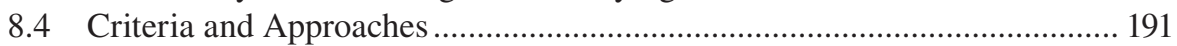

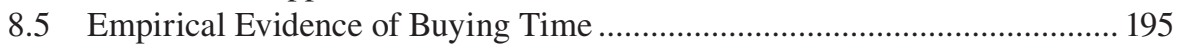

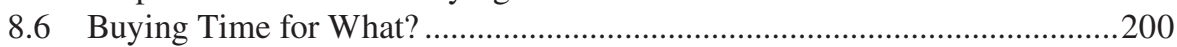

8.7 New Venues to Test for Buying Time ............................................................ 201

8.7.1 Evaluating the Pattern of Buying Time Using Field

Observations and Common Garden Experiments .............................202

8.7.2 Establishing a Causal Link between Plasticity and Persistence Using Reciprocal Transplant Experiments ........................................202

8.7.3 Assessing the Consequences of Buying Time: Does Plasticity Buy Time for Populations to Adapt to Urbanization? ………….......202

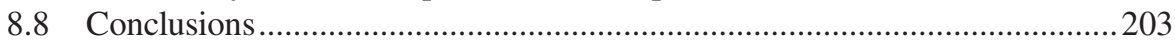

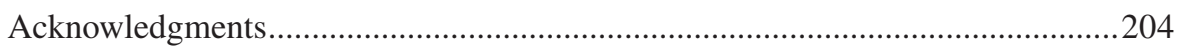

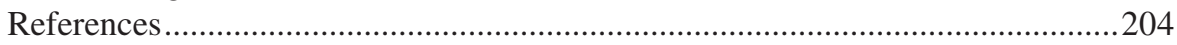

\subsection{INTRODUCTION}

As the environment changes, populations can respond by adapting via evolutionary change. If the rate or magnitude of environmental change is too great for evolution to keep pace, then populations face the risk of extinction (Chevin et al. 2010; Sinervo et al. 2010; Radchuk et al. 2019). While the majority of species that have ever lived have gone extinct, the wealth of extant biodiversity on Earth proves that many lineages have successfully survived and diversified in response to the extensive environmental challenges thrown at them since life began (see Lister 2021 in this volume).

In this chapter, we will evaluate the important role phenotypic plasticity-the ability of a single genotype to produce different phenotypes in response to environmental variation - may play in 'buying time' for populations to persist and potentially then evolve when confronted with rapidly changing or novel environments. We briefly describe the history of this concept and review the theoretical predictions 
regarding when phenotypic plasticity will or will not be able to buffer populations in changing and novel environments. We also review the empirical evidence for plasticity buying time and lay out a framework for future tests focusing on organismal responses to novel urban environments. Finally, we discuss the open questions and future directions for further research.

\subsection{A BRIEF HISTORY OF BUYING TIME}

Populations can evolve at a remarkably fast pace. Although for a long time, the action of natural selection was thought too slow to be readily observed over the course of a human life, in fact, evolution often occurs at the same timescale as ecological change (Reznick et al. 2019). Even so, adaptive evolutionary change can still be constrained for many reasons (e.g., through a lack of heritable variation, environmental stochasticity, and costs of selection). And, evidence suggests that many species are not evolving fast enough to keep pace with the current rate and magnitude of environmental change caused by a warming climate, habitat alteration, species invasions, and other anthropogenic forces (e.g., Radchuk et al. 2019). For some populations, plastic responses to these environmental challenges might be able to buffer populations from extinction, allowing time for adaptive evolution (Merilä and Hendry 2014; Diamond and Martin 2016; Fox et al. 2019).

The idea that phenotypic plasticity might buy populations the time they need to evolve (and perhaps subsequently shape evolutionary responses) is surprisingly old. Following Darwin's publication of The Origin of Species, one of the primary criticisms with the theory of natural selection was that the small intraspecific variations viewed as critical by Darwin were too slight for natural selection to effectively work upon (see Costa 2021 in this volume). This criticism led to the promotion of neoLamarckian theories of evolution, where in place of natural selection, the acquisition and inheritance of environmentally induced traits was instead the major mechanism of adaptive evolution (Simpson 1953; Crispo et al. 2010; see Bonduriansky 2021 in this volume). As a counterweight, several scientists independently proposed theories incorporating the role of environmentally sensitive traits into the framework of natural selection and Darwinian evolution (Baldwin 1896; Morgan 1896; Osborn 1896). These ideas were most thoroughly developed by James Baldwin and now are collectively known as the 'Baldwin effect' (Simpson 1953; see also Futuyma 2021 and Pfennig 2021 in this volume). Baldwin proposed that through the process of 'organic selection,' plasticity allows individuals to survive in novel and changing environments. Natural selection can then act either on standing genetic variation or on novel mutations with phenotypic effects along the same direction as the plastic effects, promoting adaptation to the novel environment by the further evolution of plastic or canalized responses. While evolutionary biologists during the modern synthesis, such as G.G. Simpson, considered the Baldwin effect plausible, they also thought it to be of little general importance and often misconstrued aspects of Baldwin's theory (West-Eberhard 2003; Crispo 2007; Scheiner 2014).

After Baldwin, Gause, Schmalhausen, and Waddington developed their own distinct theories incorporating a role for plasticity in evolution (Gause 1942; Schmalhausen 1949; Waddington 1961). Both Schmalhausen and Waddington were 
key for initiating the developmental and genetic framework for understanding phenotypic plasticity. Importantly, all of these scientists saw an important role for plasticity preceding and influencing evolutionary change. For a detailed review of the overlap and distinctions among these ideas see West-Eberhard (2003), Pigliucci (2001), Crispo (2007), as well as Futuyma (2021) and Pfennig (2021) in this volume. Nevertheless, for most of their contemporaries, phenotypic plasticity was thought to play little role in evolution overall (Pigliucci 2001; West-Eberhard 2003). While Bradshaw (1965) mentioned that plasticity could help populations with limited genetic variation adapt to strong directional selection, there was otherwise little further research on plasticity's role in adaptation to novel environments until the 1980s when West-Eberhard (1989) and Wcislo (1989) reviewed and discussed behavioral plasticity's potential roles in the evolution of novel traits and in adaptation to novel environments. WestEberhard went on to develop the concepts of phenotypic accommodation (adaptive adjustment to mutational or environmental change among integrated traits via development) and genetic accommodation (adaptive evolution of novel traits induced by mutational or environmental change), building on the theories proposed by Baldwin, Waddington, and others (West-Eberhard 2003; Crispo 2007). Around the same time as these early reviews and verbal models, formal modeling approaches started to explore the evolution of adaptive plasticity (Via and Lande 1985) and the effects of plasticity on the speed of adaptive evolution (Hinton and Nowlan 1987).

\subsection{THE THEORY AND MODELING BEHIND BUYING TIME}

From modeling and theory, how does plasticity buy time for evolution, and what are the conditions under which this will occur? Imagine a population exposed to a novel environment. If the population's mean phenotype has low fitness in this novel environment, and is not plastic, then the population faces the risk of extinction. However, if fitness-determining traits are plastic in response to the environmental change, then this plasticity could: (a) further push the population away from the new fitness optimum; (b) move the population closer to the new fitness optimum; or (c) place the population directly upon the peak (Figure 8.1). In cases (b) and (c), plasticity in the novel environment can promote population persistence and reduce the intensity of selection (Ghalambor et al. 2007). But what other factors influence the fitness effects of plasticity in novel environments and its impact on future evolutionary change? Mathematical and simulation models provide answers and testable predictions to these questions. The first model to explore this question asked if learning (a common form of plasticity in animals) could accelerate evolution. In this model, only a single phenotype conferred adaptation, while all other phenotypes were equally maladaptive, meaning that there was no slope of increasing fitness approaching the peak in the absence of plasticity. From this model, Hinton and Nowlan (1987) found that learning greatly accelerated the adaptive evolution of the population. Simply put, in this and other models of non-evolving plasticity, phenotypic plasticity smoothed out the fitness landscape by allowing individual genotypes to explore more of the phenotype space. As a consequence, less fit genotypes are able to survive and, in the presence of genetic variation, evolve towards the fitness optimum (Figure 8.2; reviewed in Frank 2011). 
(a)

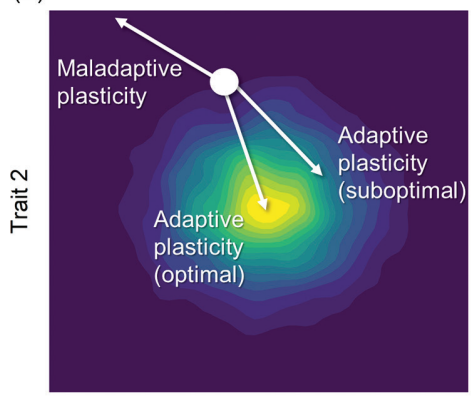

Trait 1
Fitness (b)
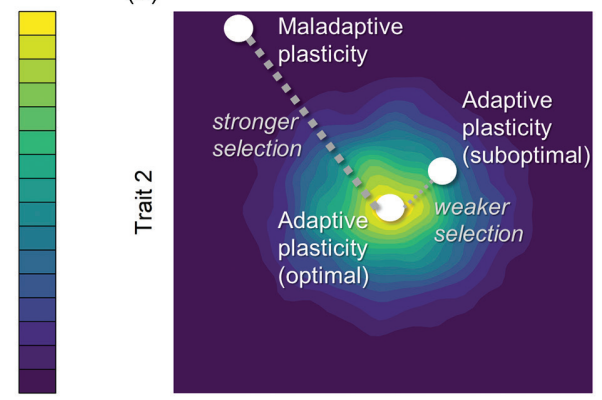

Trait 1
Fitness

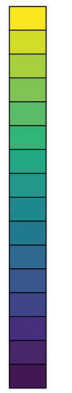

FIGURE 8.1 Variation in plastic responses to environmental change in a novel environment. In both panels, a fitness landscape for two traits is depicted, with the fitness peak in the center of the heatmap plot (and in warmer colors). (a) The population's mean phenotype (represented by the point) begins off its fitness optimum in the novel environment. Three different scenarios for plasticity are shown, including optimal adaptive plasticity that can return the population to its fitness peak; suboptimal adaptive plasticity that increases fitness but does not get the population to its fitness peak; and maladaptive plasticity that moves the population farther away from its fitness peak. (b) Consequences of the evolutionary response for different types of plasticity. Populations begin at their respective points following different forms of plasticity, i.e., the arrowheads from panel (a). Selection is weakest for optimal adaptive plasticity as there is no variation to act upon; selection is weak, but present for suboptimal adaptive plasticity; and selection is strongest in the case of maladaptive plasticity. Modified from Ghalambor et al. (2007).

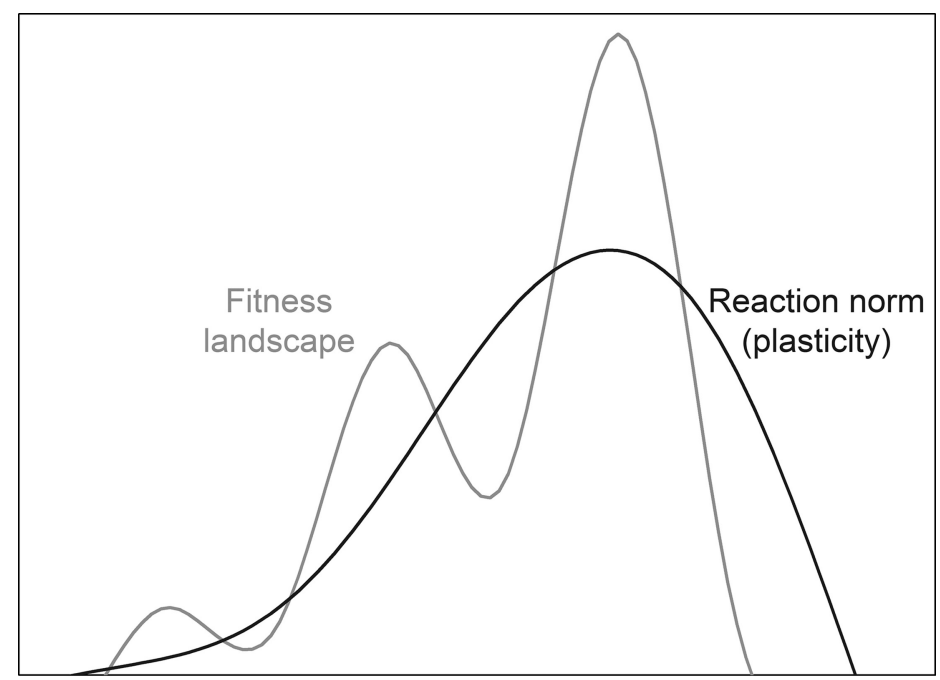

Phenotype

FIGURE 8.2 A high degree of plasticity results in smoothing of a bumpy, multi-peaked fitness landscape. Redrawn from Frank (2011). 
However, plasticity can also slow the rate of evolution and negatively impact persistence (Ancel 2000; Ghalambor et al. 2007; Paenke et al. 2007). For plasticity to facilitate evolution and persistence, plasticity must be somewhat adaptive in the new environment, although perfect plasticity that matches the fitness optimum will increase persistence but prevent further evolution (Figure 8.1; Price et al. 2003; Ghalambor et al. 2007; Paenke et al. 2007). Moreover, the effects of plasticity on evolution depend on which genotypes benefit. Plasticity positively affecting relatively more-fit genotypes will generally speed evolution but evolution will be impeded if instead less-fit genotypes are more plastic (Paenke et al. 2007).

In general, the models discussed above assume an unchanging environment, do not model population persistence directly, and omit potential costs of plasticity. Using a model incorporating a continuously changing environment and costs to plasticity, Chevin et al. (2010) found that adaptive plasticity did enable population persistence, although it also reduced the strength of directional selection. Moreover, they found that costs of plasticity constrained the population's ability to track environmental change and that persistence was greatest with intermediate levels of plasticity (Figure 8.3; also see Nunney 2016). Building further upon these models, Reed and colleagues (2010) modeled plasticity's ability to buffer populations in response to a temporally fluctuating environment. Here, they found that when environmental stochasticity was moderate and predictable (i.e., by an environmental cue), even weakly adaptive plasticity could buffer populations and allow them to persist. However, when environmental stochasticity was high and unpredictable, plasticity resulted in a greater phenotype-to-environment mismatch, and increasing the magnitude of plasticity could even increase the risk of extinction.

The models described above show that, as long as plasticity is somewhat adaptive in the new environment, plasticity can buy time for populations to evolve under some conditions. However, when genetic variation in reaction norms exist, plasticity itself can evolve (Scheiner 1993), creating a dynamic interplay between plasticity and evolution. How does the evolution of plasticity affect its ability to buffer populations from the impact of environmental change?

Verbal models (e.g., Baldwin 1896; Waddington 1961; West-Eberhard 2003) suggest that the evolution of plasticity can speed the rate of adaptation to novel environments (reviewed in Price et al. 2003; Ghalambor et al. 2007), and recent formal modeling approaches have been useful in identifying under what conditions this can occur. There is broad consensus among these models that phenotypic plasticity, when there is little associated cost, significantly increases population persistence in novel and changing environments, and that allowing plasticity to evolve enhances this effect and speeds up the rate of adaptation (Lande 2009; Chevin et al. 2010; Scheiner et al. 2017, 2020). This is true whether environmental change is modeled as an abrupt shift (with small temporal variability before and after) or as a directionally moving optimum. Moreover, models show that plasticity, and the evolution of plasticity, can also facilitate persistence and adaptation to new environments even in the presence of gene flow from source populations (Chevin and Lande 2011; Thibert-Plante and Hendry 2011). Altering the predictability of environmental change however can limit the ability of populations to persist, even with evolving plasticity (Ashander et al. 2016). And when costs of plasticity are 


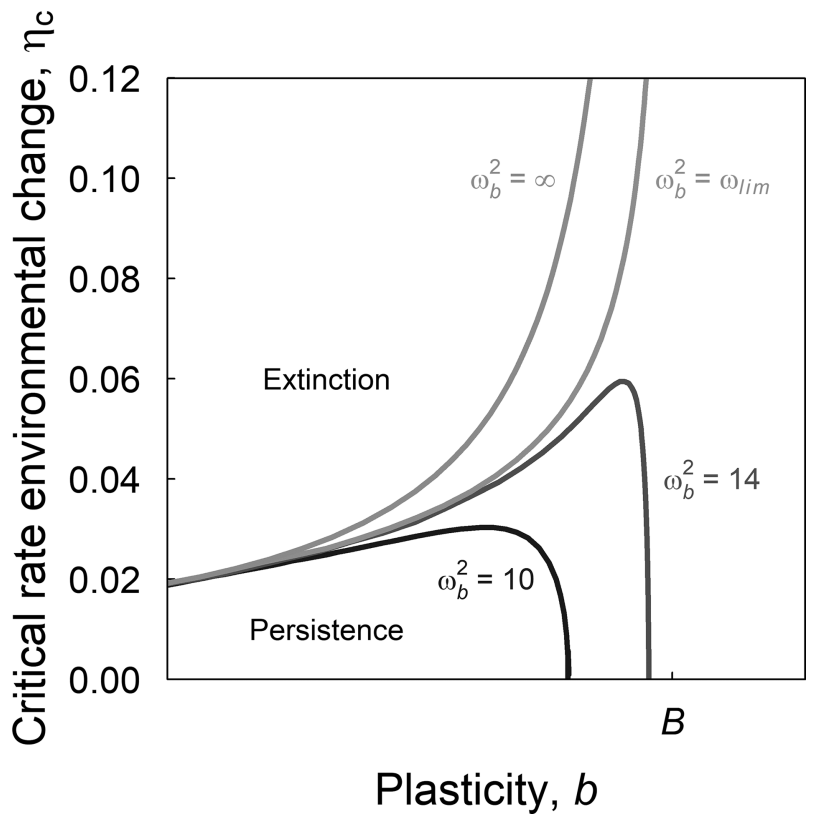

FIGURE 8.3 Results from a model showing that the critical rate of environmental change depends on the cost of phenotypic plasticity. The maximum rate of environmental change that supports long-term persistence of a population, $\eta_{c}$, is expressed as a function of plasticity, $b$, at four values of the cost of plasticity, $\omega_{\mathrm{b}}^{2}$. Increasing costs of plasticity are indicated by smaller values of $\omega_{\mathrm{b}}^{2}$, the width of the fitness function for plasticity. In this example, $\omega_{\mathrm{b}}^{2}$ includes values of 10 (the highest cost of plasticity considered), 14 (a comparatively lower cost of plasticity), $\omega_{\lim }$ (the threshold for the cost of plasticity), and infinity (no cost of plasticity). Rates of environmental change higher than each line indicate population extinction, and rates of environmental change below each line indicate population persistence. The populations with relatively high costs of plasticity, that is, when $\omega_{\mathrm{b}}^{2}=10$ and when $\omega_{\mathrm{b}}^{2}=14$, only persist at relatively low values of plasticity, $b$ (shown in the purple and blue lines). However, for these scenarios where $\omega_{\mathrm{b}}^{2}<\omega_{\lim }$, note there are medium-high values of plasticity that maximize the critical rate of environmental change. In this model, $B$ indicates the environmental sensitivity of selection. Redrawn from Chevin et al. (2010).

high (Scheiner et al. 2020) or when predictability is very low, the evolution of greater plasticity can potentially be maladaptive, increasing the risks of population extinction (also see Lande 2015).

In sum, theory suggests that in novel and changing environments, adaptive plasticity can buy time for populations to evolve, and can even speed this process, especially when plasticity itself can evolve, costs of plasticity are low, and environmental variability is predictable. However, these results also point towards the need for more empirical data to test the models' assumptions and predictions (Box 8.1). For example, when they are measured, costs of plasticity are generally small. However, these are rarely measured in novel environments where additional costs may be imposed (Chevin and Hoffmann 2017; Snell-Rood et al. 2018). 


\subsection{CRITERIA AND APPROACHES}

Several authors have enumerated aspects of the criteria and approaches for buying time (Robinson and Dukas 1999; Pigliucci 2001; Wund 2012; Morris 2014). We synthesize this previous work here to develop a unified set of criteria and review the different ways in which buying time might be assessed. Our main goal in this section is to develop a generalizable summary of criteria and approaches. In the following section, we then apply these criteria and approaches to our review and synthesis of empirical case studies of buying time.

Effectively, there are two criteria for demonstrating buying time via plasticity. First, the population must be exposed to novel environmental conditions beyond those experienced in the ancestral environment. We take a broad view of this criterion to include changes in both the frequency and magnitude of the environmental change. For example, under this definition, it would be sufficient for a population that occasionally experiences extreme high temperatures to experience them more frequently as the environment changed, even if those temperatures were not, in a strict sense, outside the actual range of temperatures experienced by the ancestral population. Of course, populations might also experience novel conditions completely outside the range of ancestral variation, such as in the case of exposure to a novel pesticide or other novel environmental toxin. Second, the population must persist under the novel environmental conditions as a direct consequence of plasticity. Because the causal relationship can be difficult to establish, weaker evidence involves demonstrating that the population has equal or greater fitness due to plasticity expressed in the novel environment. Specifically, the presence of plasticity and the demonstration of population persistence over time would be incomplete evidence of buying time via plasticity. Only through the causal association of variation in plasticity with variation in population persistence can buying time be conclusively shown. Either plasticity or population persistence on its own is insufficient, as populations might persist via other mechanisms (e.g., immigration; rapid evolution) and plasticity, while present, might be unlinked with population persistence. A third aspect of buying time via plasticity - subsequent genetic adaptation of the population, i.e., genetic accommodation-is more a consequence of buying time rather than a necessary criterion for its existence. Although, importantly, the evolution of plasticity itself can enhance its effects on population persistence (see Section 8.3).

Buying time via plasticity can be tested in a number of different ways by using laboratory and field experiments and population- and species-level comparative approaches. The most direct test of this hypothesis would be to choose replicate populations that differ in their plasticity for a single or suite of traits in a novel environment, but are otherwise matched, and assess whether the magnitude of plasticity is positively associated with persistence after being exposed to novel environmental conditions. Such an approach could be carried out in either the field or laboratory setting. However, there is likely to be a limited subset of organisms for which this approach is possible given the constraints on having sufficiently short generation times to be able to track changes in population size over reasonable timeframes. Alternatively, historical versus contemporaneous population samples can be used to test for buying time via plasticity. To establish an association between 
plasticity and persistence, at least one historical population size sample, or preferably regular monitoring of the population, must be present. This method could be less able to establish a causal link between plasticity and population persistence, as many such studies lack an experimental manipulative component and records on the nature of the precise environmental change could be sparse. Further, as in many fields of study, historical samples to match against contemporary samples and regular monitoring of populations over time tend to be relatively rare. There are, however, a number of benefits of tests of buying time via plasticity that are performed using matched historical-contemporary samples in field settings. Organisms with longer generation times can be tested, and environmental changes experienced in situ are inherently more biologically relevant.

Extant, contemporaneous population and species comparative approaches are yet another way that buying time can be tested. For example, natural variation of the reaction norms among populations of a species exposed to ancestral versus novel environmental conditions can be compared. Similarly, such approaches can be extended to higher levels of biological organization, such as species that differ in their plasticity. As with any comparative approach of this nature, increasing the level of biological organization of analysis tends to increase the potential for confounding variables. Further, like the historical-contemporary comparisons described earlier, with the use of comparative approaches on contemporaneous groups, it can be challenging both to establish a causal link between plasticity and population persistence and to disentangle current plasticity from ancestral plasticity that allowed species to persist under some previous environmental change. By contrast, the major benefit of comparative approaches lies in the large number of species that can be evaluated for the plasticity-persistence association.

In context of these different approaches to testing buying time via plasticity, it is worth considering what types of traits should be examined. As a general rule, examining plasticity in traits that determine fitness should be preferred, since the ability of a population to maintain high mean population fitness while experiencing changing environmental conditions is the core tenet of this hypothesis (Lande 2014). Indeed, while there should be minimal plastic variation in fitness across ancestral and novel environments, plasticity in traits such as morphology, behavior, and physiology will ideally allow populations to maintain high fitness when experiencing environmental change. Depending on the type of trait and nature of the plasticity, greater plasticity can manifest as, for example, a steeper slope in the relationship between the trait value and the environment, or as a broader curve over a range of phenotypic values. Alternatively, trait values might be fixed across ancestral and novel environments via trait canalization to maintain high fitness under changing environmental conditions; however, such fixation of traits across environments can be achieved via plasticity in other traits (Grether 2005).

Beyond demonstrating the ability of plasticity to promote population persistence, studies should also aim to test specific predictions from theory to evaluate the conditions in which buying time will occur. For example, models suggest that costs of plasticity, the predictability of environmental variation, and the covariance of plasticity and fitness affect the likelihood that plasticity will rescue populations (see Section 8.3). These parameters can be measured in natural systems and model 
systems of experimental evolution could be well-suited for manipulating these effects. In addition, different mechanisms of phenotypic plasticity have themselves evolved in response to these same conditions, suggesting that some mechanisms of plasticity may be more likely to buy time than others in novel environments.

Recently, Snell-Rood et al. (2018) formalized definitions of three general types of plastic response to changing environmental conditions including: developmental selection, generalized physiological responses, and phenotypic switches. These different plastic mechanisms carry different costs to produce the phenotype and confer different likelihoods that the phenotype will be adaptive in a novel environment (Figure 8.4; see also Lande 2014). While developmental selection carries the highest cost of phenotype, it is also the most likely to be adaptive in the novel environment. Developmental selection mechanisms encompass environment-induced changes in learning, acquired immunity, tissue architecture and the microbiome. As a consequence, developmental selection mechanisms are highly targeted to the novel environment but are costly because they require considerable investment throughout development in response to feedback from the environment. Generalized physiological responses are intermediate with respect to both cost of phenotype and the likelihood of being adaptive in the novel environment. Such generalized physiological responses are not specifically targeted to the new environment, so there can be a waste of resources into broad stress responses that might not cover all possible stressors which increase the cost of phenotype. However, there is often cross-protection from generalized physiological stress responses that can increase the likelihood of being adaptive in the novel environment: for example, increased heat shock proteins can protect against both heat and desiccation stress (Chown and Nicolson 2004). Finally, phenotypic switches, while carrying low costs of phenotype, are the least likely to be adaptive in the novel environment. Phenotypic switches are some of the most commonly described forms of plasticity and encompass polyphenisms and graded developmental responses. Because these responses are highly tailored, the likelihood that phenotypic switches will be adaptive in the novel environment is quite low. However, this expectation does not preclude the fact that there might still be a relatively small subset of environmental changes that allow such phenotypic switches to preadapt organisms for a given novel environment. Although any one of these plastic mechanisms can buy time for populations experiencing environmental change, this framework allows us to develop predictions for their ability to match and be adaptive in the novel environment against the costs required to produce these phenotypes.

In a similar vein, whether plasticity is adaptive in the novel environment will also depend on the nature of the environmental change. Assuming that plasticity evolved in the ancestral environment in response to a specific set of environmental conditions, if the novel environment is an extension of the ancestral environment, such as an increase in environmental temperature, then the plastic response could be somewhat adaptive in the novel environment. However, if the novel environment is either characterized by an extreme shift well beyond the range of the ancestral environment or truly novel (i.e., an environment never experienced before over the evolutionary history of the species, such as a novel environmental toxin), then it is much less likely that plasticity will be adaptive in the new environment. Although importantly, these 


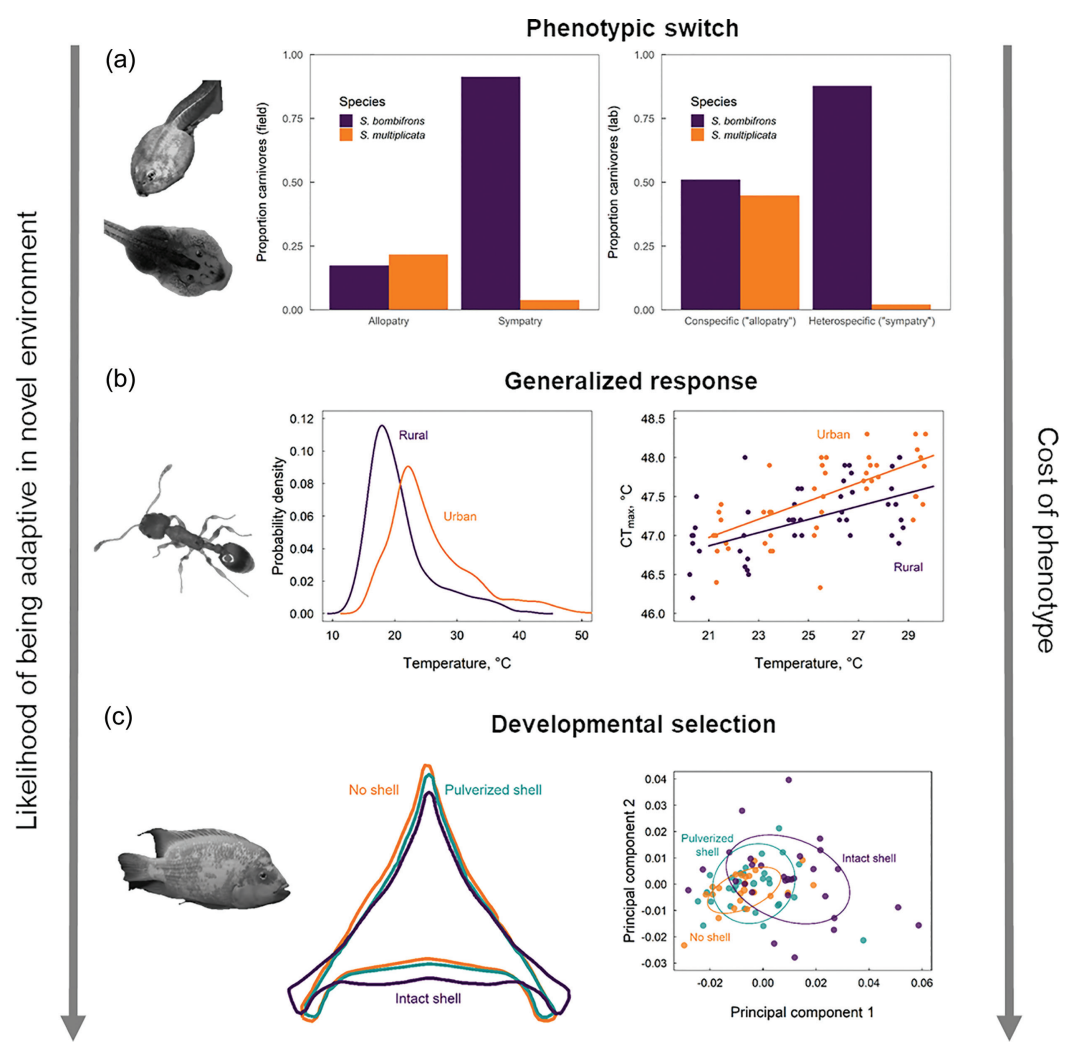

FIGURE 8.4 Case studies of different types of plasticity rescue expressed as functions of their likelihood of being adaptive in the novel environment and the cost of phenotype (sensu Snell-Rood et al. 2018). (a) Spadefoot toad tadpoles (Spea spp.) exemplify a phenotypic switch, with a low likelihood of being adaptive in a novel environment but a low cost of phenotype. In this system, consumption of shrimp or other tadpoles induces a developmental switch from a typical omnivore morph to a carnivore morph. Under allopatric conditions in the field or reared with conspecifics in the lab, S. multiplicata and S. bombifrons produce carnivores and omnivores in similar ratios. However, under sympatric conditions in the field or reared with heterospecifics in the lab, S. bombifrons adaptively produces a high proportion of carnivores whereas $S$. multiplicata adaptively produces almost none, reducing interspecific competition [data from Pfennig and Pfennig (2012).] (b) Acorn ants exemplify a generalized response, with intermediate likelihood of being adaptive in a novel environment and an intermediate cost of phenotype. In this system, a plastic increase in heat tolerance (the critical thermal maximum, $\mathrm{CT}_{\max }$ ) allows acorn ants to cope with elevated temperatures and might have allowed rural population acorn ants to persist until the evolution of higher heat tolerance could occur [data from Diamond et al. (2018).] (c) The Midas cichlid exemplifies developmental selection, with a high likelihood of being adaptive in a novel environment but a high cost of phenotype. Fish that were fed on snails with their shells intact had shorter, less concave jaws with broader, more outwardly pointing horns (towards the base of the jaw) compared with fish fed on either standard fish food or snails with pulverized shells [data from Muschick et al. (2011)]. Photo credits (grayscale of originals): spadefoot toad tadpoles: David Sanders; acorn ant: Ryan Martin; Midas cichlid: Mahufi/Wikimedia Commons. 


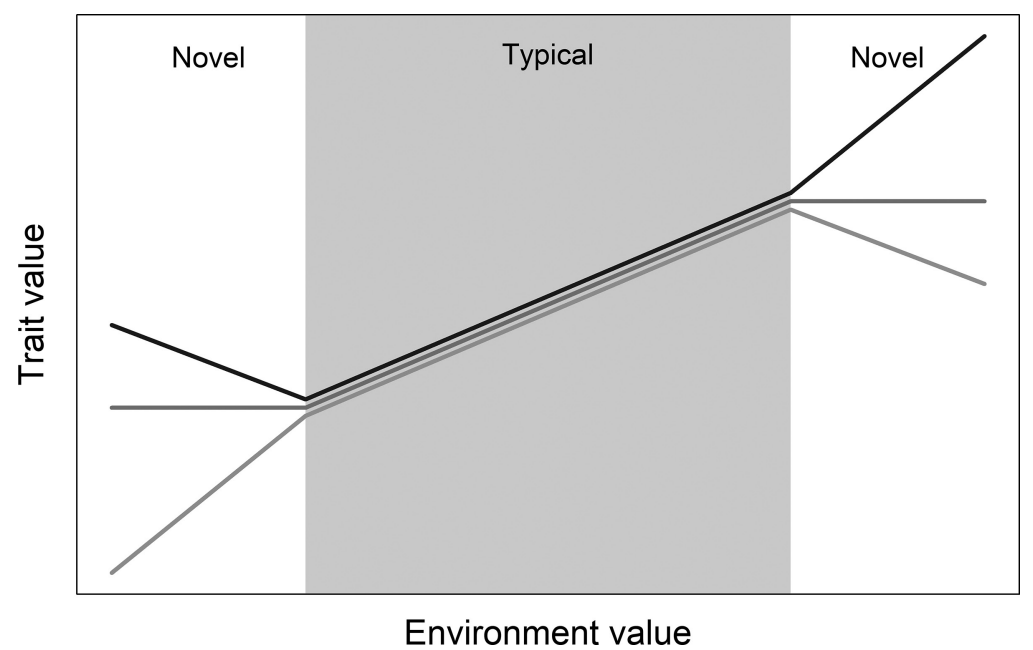

FIGURE 8.5 Reaction norms of three genotypes under typical and novel environmental conditions. Genotypes have similar responses to environmental variation under typical conditions. By contrast, genotypes have divergent responses to novel environmental conditions, under both lower and higher than usual environment values. (Redrawn from Ghalambor et al. [2007].)

novel environmental conditions could also expose cryptic heritable variation in the reaction norm (Figure 8.5), potentially allowing for adaptive plasticity to quickly evolve (Ghalambor et al. 2007).

\subsection{EMPIRICAL EVIDENCE OF BUYING TIME}

In this section, we discuss the currently available evidence for buying time via plasticity. However, first we aim to direct readers to reviews of buying time via plasticity. Representative studies can be found throughout more general compilations such as West-Eberhard (2003), and within specific sections on the topic of buying time of many additional works (Robinson and Dukas 1999; Pigliucci 2001; Ghalambor et al. 2007; Wund 2012; Morris 2014; Schlichting and Wund 2014; Fox et al. 2019).

We begin by highlighting strong examples of buying time, specifically those that either directly meet both criteria for buying time, or at least, have strongly suggestive data. These studies show evidence of populations experiencing changing and novel environmental conditions and establish a strong link between plasticity and population persistence. We then highlight related fields of study that provide other examples of buying time, including the literature on genetic accommodation, invasion biology, biological responses to global climate change, and recent work on plasticity of the microbiome.

Perhaps the most complete example of buying time via plasticity is that of plastic changes in breeding phenology that allow dark-eyed juncos from a temperate, montane habitat to establish in a lowland, coastal habitat (Yeh and Price 2004). This example of buying time is especially strong, as the authors were able to pinpoint the 
actual colonization of the coastal habitat in the early 1980s and were able to monitor population size for six years after the introduction, from 1998 to 2003. Further, the ability to plastically extend the breeding season in the coastal habitat was associated with the maintenance of population size over the study period, strongly suggesting a link between the plasticity and population persistence without evolutionary change.

Alternative phenotypes can also be important types of plasticity that buy time for populations in novel environment conditions. Ecological character displacement in two species of spadefoot toad tadpoles is a prime example. In the American Southwest, Spea bombifrons and S. multiplicata produce approximately equal ratios of two environmentally induced phenotypes when found alone in allopatry. The two phenotypes represent a developmental switch to a carnivore morph with large jaw muscles, serrated beaks, and shortened intestines that allows them to specialize on larger prey such as fairy shrimp and tadpoles, from an omnivore morph that lacks these features and feeds primarily on algae and detritus. However, in sympatric ponds, there are species-specific shifts in the ratios of these two morphs. S. bombifrons produces mostly carnivores whereas $S$. multiplicata produces mostly omnivores (Figure 8.4a; Pfennig and Murphy 2000, 2002). By being able to modulate this developmental switch, the species reduce competition for food which, in turn, facilitates persistence (Pfennig et al. 2006, 2007). Further, in S. multiplicata, the differences in the production of carnivore morphs between allopatric and sympatric populations appear to be mediated by plasticity via a condition-dependent maternal effect (Pfennig and Martin 2009; Martin and Pfennig 2010; Levis and Pfennig 2019).

Despite their specificity (Snell-Rood et al. 2018), alternative phenotypes under the control of developmental switches may be an effective means of buying time in situations where preexisting plasticity is immediately adaptive in novel environments because the selective pressure, although novel, is similar in action to a fluctuating selective pressure in the ancestral environment (West-Eberhard 1989, 2003; e.g., gain or loss of predators, competitors or prey). Indeed, such developmental switches based on resource-use traits appear to be a highly repeatable form of buying time via plasticity. Pfennig and McGee (2010) used a series of replicated sister-group comparisons in spadefoot toads as well as centrarchid fish, salmonid fish, neotropical cichlids, and mole salamanders to show that plasticity in resource use and the consequent developmental switch was positively associated with geographic range size and species richness for a given clade, two proxies of persistence.

Comparative approaches of a similar nature, though without relying on sistergroup comparisons, have also recently been used to test for buying time via plasticity across a diverse suite of bird species. Ducatez et al. (2020) performed a meta-analysis of over 8000 species of birds and over 3800 novel behaviors and found that those species with a higher propensity to innovate were at a lower risk of global extinction and are more likely to have increasing or stable populations than less innovative birds (see also Sol et al. 2002, 2005). Studies of this nature, while less able to establish direct causation between plasticity and persistence, nonetheless suggest that this mechanism could be fairly widespread.

Experimental transplantation studies also provide evidence for plasticity promoting persistence in novel environments. For example, Volis et al. $(2014,2015)$ transplanted wild emmer wheat from four natural populations into a low rainfall desert site, outside 
the current environmental conditions for the species. Using plasticity measured in a separate greenhouse experiment with drought and normal water conditions, they found that the most plastic population was also the most successful, measured by estimated lifetime fitness, in the novel desert environment (see Donohue et al. 2001 for another example). In a related phenomenon, the 'polyploid plasticity hypothesis' suggests that the widespread success of polyploid plant species (and other taxa) at colonizing novel and diverse habitats, in comparison to closely related diploid species, is due in part to a greater propensity for plasticity in polyploids (Levin 1983; Van de Peer et al. 2017). However, the status of this hypothesis is currently unclear with both some positive (e.g., Hahn et al. 2012) and negative (e.g., Wei et al. 2019) support for the role of plasticity in the success of polyploids across diverse environments.

In addition to these examples, other likely places to look for buying time comes from the genetic accommodation literature. As genetic accommodation describes the process by which newly exposed plasticity (or other novel traits) are subsequently refined by selection, such studies are likely to contain not only the after-effects of buying time, but varying degrees of evidence of the process itself, namely the link between plasticity and population persistence. For example, Badyaev (2009) summarized the Baldwin effect processes operating in the rapid range expansion and colonization of novel habitats across North America by the house finch. Novel high temperatures at the southern range edge and novel cool temperatures at the northern latitude range edge exceed egg-viability limits in the expanding range edge populations. These conditions induce novel behavioral and physiological variation including changes to the traits of environmental assessment, incubation behavior, and oogenesis. Because these traits are all underlain by a common hormonal pathway, they shift in concert in response to environmental change. Female finches can alter offspring size via shifts in hatching order which, in turn, determine offspring growth rate. At the northern range edge, small males and large females are favored and breeding females produce daughters in first-laid eggs and sons in last-laid eggs. However, at the southern range edge, large males are favored, and breeding females produce sons in the first-laid eggs and daughters in the last-laid eggs. As a consequence, the direction of sexual size dimorphism is opposite at the expanding northern and southern range edges. Following induced plasticity in egg laying order, natural selection has acted to refine this variation to maintain reproductive homeostasis under novel environmental conditions. As part of this study, the finch populations were monitored over a severalyear period, allowing the researchers to establish a fairly strong association between the egg laying plasticity and the maintenance of population size over time.

Other genetic accommodation papers lack such population persistence data, but nonetheless represent plausible cases where plasticity buys time for evolution to occur. For example, Hua et al. (2015) found that plasticity in the ability to tolerate elevated concentrations of the insecticide, carbaryl, likely facilitated the evolution of insecticide tolerance in wood frog populations. As selection continued to refine the tolerance plasticity, the plastic variation was lost (i.e., the trait was canalized) in populations with high exposure to the insecticide. By contrast, populations with minimal exposure to the insecticide retained plasticity in tolerance. Although population sizes were not directly monitored as part of this study, the fact that initial plasticity was acted upon by natural selection and populations remain extant are suggestive of 
plasticity buying time in this system. Similarly, Corl et al. (2018) found evidence of evolutionary divergence in the coloration of side-blotched lizards across light-colored sand habitats and dark-colored lava flow habitats, and that this divergence was likely promoted by plasticity in body coloration in response to the background coloration of the habitat. Shifts in the resource base can also drive the genetic accommodation process. Aubret and Shine (2009) document evolutionary divergence in the head size of tiger snakes across different islands. The islands harbor populations of snakes that differ in their time since colonization of the habitat, and such differences are associated with evolutionary divergence in their responses to prey. The older island populations have become canalized for larger head size. This response appears to be facilitated by a plastic morphological response to variation in prey size, as the newer island population snakes have relatively small head size at birth, but plastically increase their head size in response to large prey items. Finally, tests of the flexible stem hypothesis (West-Eberhard 2003), which predicts that the diverse phenotypes within an adaptive radiation emerge from ancestral developmental plasticity, provide evidence of plasticity's importance in the adaptation to novel environments. Diverse examples, including sticklebacks (Wund et al. 2008), Anolis lizards (Losos et al. 2000; Kolbe and Losos 2005) and spadefoot toads (Gomez-Mestre and Buchholz 2006; Kulkarni et al. 2017) match this pattern. Similarly, West-Eberhard (2003) hypothesized that the repeated, independent evolution of crassulacean acid metabolism (CAM) photosynthesis in plants in response to environmental stress was facilitated by widespread ancestral plasticity in photosynthetic pathways.

Invasion biology is another, if less obvious, place to look for evidence of buying time. Greater plasticity of invasive species has been hypothesized as a key mechanism underlying their success (Baker 1965). This idea has been especially well-tested in plants, and while there does seem to be some positive support for a link between persistence of invasive species in novel habitats and plasticity, a number of counterexamples exist (reviewed in Richards et al. 2006). Davidson and colleagues (2011) performed a large comparative study of phenotypic plasticity in 75 invasive versus non-invasive plant species pairs. They found that while invasive species harbored greater plasticity, it was the non-invasive species that maintained greater fitness homeostasis, potentially maintaining their population sizes over time. However, individual studies do provide some evidence of a plasticity-persistence link. For example, Funk (2008) found evidence that plasticity in plant functional traits was higher in invasive plant species compared with non-invasive species pairs among a diverse group of plant taxa in Hawaii. These traits were strongly associated with plant performance and fitness suggesting that such plasticity could aid the persistence of the invasive species in the novel habitat. Similar results were found in native-introduced range population comparative study of an Asian annual plant recently introduced to North America (Matesanz et al. 2012). On the other hand, plasticity may buffer native species from invaders. Trussell and Smith (2000) found that a rapid increase in shell thickness in an intertidal snail during the early 20th century was in fact due to adaptive plasticity that may have quickly evolved in response to an introduced crab. It is unclear if this plasticity was present before the predator invasion, but the response may have evolved as adaptive plasticity rather than a canalized change due to the large cost of shell thickening on body mass (Trussell and Smith 2000). 
Arthropod systems provide further support of a plasticity-persistence link in the context of invasion biology. For example, the invasive Mediterranean fruit fly Ceratitis capitata has much greater acute thermal tolerance plasticity compared with its native congener $C$. $\operatorname{ros} a$, and this difference in plasticity is associated with a much larger geographic range of $C$. capitata compared with $C$. rosa (Nyamukondiwa et al. 2010). In addition, Chown et al. (2007) examined plasticity in desiccation tolerance in response to chronic and acute temperature treatments among native and invasive springtail species. While they did not find plastic differences in the absolute limits of tolerance between the species, they did find that the shape of the plasticity differed between the species. In particular, the invasive species were consistent with a 'hotter is better' relationship whereas the native species were consistent with a 'colder is better' relationship for desiccation tolerance. Under global climate change, the invasive species were predicted to have higher population persistence.

Indeed, the literature on biological responses to climate change is likely to be another fruitful area for exploring buying time via plasticity. In addition to the springtail system described above, tolerance plasticity appears to be a common feature of many ectothermic species, and while the magnitude of plasticity is, on its own, generally insufficient to cope with global climate change (Gunderson and Stillman 2015; Sørensen et al. 2016), it could still buy time for populations to evolve. Similar scenarios are unfolding for other aspects of global change including alterations to natural habitats for agriculture and human settlement. Recent work in Anolis (Campbell-Staton et al. 2020) and Leiocephalus (Neel et al. 2020) lizard species suggests that plasticity has been important for the establishment of populations within heavily urbanized areas. Transgenerational plasticity could likewise facilitate persistence, although importantly, such a mechanism relies on the reliable environmental cues (Donelson et al. 2018). For example, a long-term study on blue tits found that the expected temporal trend of earlier laying dates was only detected in populations inhabiting evergreen forests, but not in nearby deciduous forests. Laying date phenology (a maternal effect) is critical in this system for being able to track the timing of peak caterpillar prey abundance. The divergence in the evergreen versus deciduous forest responses was driven by the fact that climatic warming was greatest during the spring and minimal during the winter. The evergreen forest populations have cue windows during a short time in the spring whereas the deciduous forest populations have a very broad cue window spanning the winter and part of the spring (Bonamour et al. 2019).

Finally, some of the newest research areas that could be used to test the buying time hypothesis involve plasticity in the microbiome. The underlying idea is that organisms, by changing the composition or expression of their microbiome, can alter their (extended) phenotype (i.e., plasticity) to respond to novel environments (Alberdi et al. 2016). For example, naive Drosophila switched to a novel high-salt diet performed better and were able to persist when given the gut microbiome from flies already experimentally evolved on the resource (Markov and Ivnitsky 2016). Although the microbiome represents a relatively new research frontier and there are few studies to summarize at this point, there is still high potential for the microbiome to serve as an important mechanism mediating the buying time response and allowing populations to cope with environmental novelty (reviewed in Alberdi et al. 2016). 
Up to this point, we considered studies that provided positive support for the buying time hypothesis. However, it is worth discussing examples where plasticity failed to buy time. A prominent example is the work of Sinervo et al. (2010) in which, despite having plasticity in thermoregulatory behavior to avoid overheating, many lizard populations in Mexico were found to have gone extinct under recent climate change. In this case, the plasticity was in an adaptive direction to avoid overheating but restricted the available foraging times for lizards. As a consequence, thermoregulatory plasticity was unable to rescue the lizard populations outright, nor to buy time for the populations to adapt. In fact, the genetic architecture of the thermoregulatory plasticity and other thermal traits further limited adaptation. Of course, one could argue that the plasticity still bought some time for the lizards, just not enough time. Another example, also in lizards, comes from Telemeco et al. (2017). In this system, Sceloporus lizards historically adjusted the depth of their nests based on environmental temperature, such that warmer temperatures would elicit shallower nest depths, and cooler temperatures would elicit deeper nest depths as a protection against cold extremes. However, with climate change the thermal plasticity in nest depth has become maladaptive, as shallower nests under warmer conditions now place the developing lizard eggs at risk of heat stress. Thus, plasticity is potentially hastening vulnerability to environmental change rather than buying time against it.

\subsection{BUYING TIME FOR WHAT?}

Arguably, one of plasticity's most important roles is to buy time for populations to adapt evolutionarily, i.e., to undergo heritable changes that increase fitness and thus persistence in the new environment. The conditions under which plasticity is able to rescue populations outright appear to be quite limited, either due to an insufficient magnitude of plastic response to fully cope with environmental change or the fact that plasticity will not necessarily be adaptive in novel environments. Such limitations are magnified in light of recent global change processes, characterized by unprecedented shifts in the environments that most organisms inhabit. Thus, the combination of both plastic and evolutionary mechanisms, and in particular, plasticity's role as a means of buying time for evolution to occur, might be expected to be critical for rescue and persistence of many populations experiencing environmental change (Diamond and Martin 2016).

Although our primary focus is on buying time for populations, potentially with the second step that evolutionary change will have sufficient time to take place, we think it is important to also address the fact that plasticity can likewise hinder evolution. In this case, plasticity technically buys time for populations, but rather than allowing sufficient time for evolution to occur, it shields variation from selection and limits evolutionary change. For example, in Anolis lizards, behavioral shifts to using microclimates on tops of boulders at high altitude allow thermoregulation via basking in these cool environments; however, the thermoregulatory behavior shields variation in thermal performance from selection (Muñoz and Losos 2017). And as environmental temperatures continue to increase, the buffering effect of such thermoregulation will likely be insufficient to prevent eventual population extinction (sensu Sinervo et al. 2010). If there is heritable variation for the plastic trait itself, or if cryptic genetic variation is released in novel environments (Figure 8.5), then the 
evolution of plasticity may be able to rescue populations from these types of evolutionary traps (Diamond and Martin 2020). However, evolving increased plasticity in changing environments can carry its own risks as models have found that this itself can lead to extinction under some conditions (Scheiner et al. 2017, 2020).

\subsection{NEW VENUES TO TEST FOR BUYING TIME}

Although evidence of buying is accumulating, many outstanding questions on the prevalence and mechanisms of buying time remain (Box 8.1). Indeed, while a number of different types of approaches have been used to test for buying time via plasticity, there are still untapped outlets for evaluating this hypothesis. Cities represent an excellent setting in which buying time via plasticity can be tested, as they are often typified by substantial environmental change over a short spatio-temporal range (Rivkin et al. 2019). This property permits the assessment of contemporary population persistence via plasticity. Cities might act as environmental filters which plasticity could overcome (Figure 8.6a). Plasticity could also allow persistence of these newly immigrated individuals or resident urban populations (Figure 8.6b). Both mechanisms would have the same general outcome with respect to the variation of plasticity in the population. In the ancestral environment, a range of plastic genotypes might be supported (Figure 8.6c), whereas in the novel, urbanized environment, only the most (adaptively) plastic genotypes are able to persist (Figure 8.6d). Below, we outline a research program that uses cities as a tool to explore the buying time hypothesis.

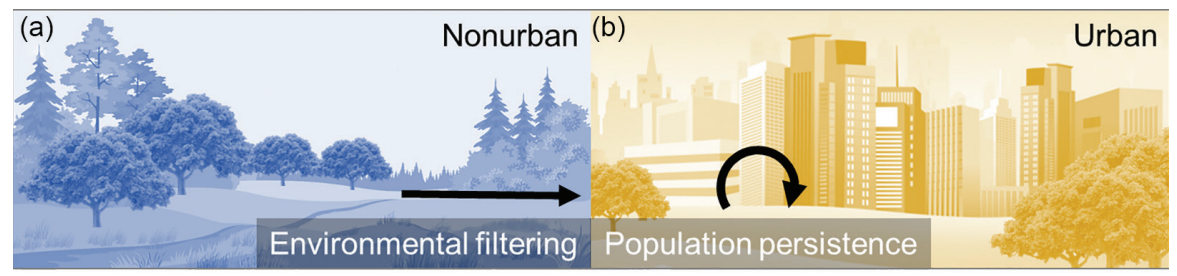

(c)

(d)
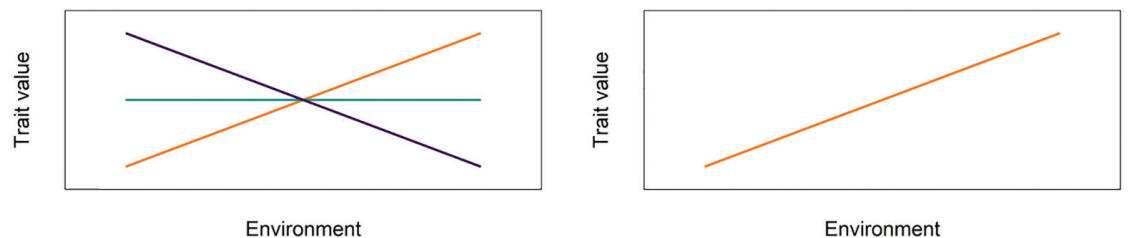

FIGURE 8.6 Patterns and processes of how plasticity might allow organisms to live in human-altered urbanized environments. (a) Plastic phenotypes are those that can traverse the boundary from nonurban environments to urban environments via environmental filtering. (b) Plasticity might allow population persistence of organisms already in urbanized environments. (c) Reaction norms of three genotypes, including one with adaptive plasticity (positive slope, orange line), no plasticity (zero slope, green line), and maladaptive plasticity (negative slope, purple line) in the novel, urban environment. Nonurban environments might be expected to harbor all types of reaction norms. (d) Only the reaction norm exhibiting adaptive plasticity (positive slope, orange line) either enters or persists in the urbanized environment. 


\subsubsection{Evaluating the Pattern of Buying Time Using Field Observations and Common Garden Experiments}

Through already-available and new surveys, species can be identified that either occur only in nonurban habitats or occur in both nonurban and urban habitats. These species can be collected from the nonurban habitat and reared under common garden conditions until a new generation of individuals is produced. Species can be reared under two or more environmental conditions to assess developmental plasticity, or species can be reared under a single environment and assessed for acute plasticity. Common urban stressors include temperature, aridity, environmental toxins, and sound/light pollution, which could serve as the environmental treatments to assess plasticity. Following this list of stressors, relevant traits would then include thermal tolerance, desiccation tolerance, and toxin tolerance. If plasticity buys time for urban populations, then the steepness of the reaction norm or the width of the tolerance/performance curve should be positively associated with the presence across both urban and nonurban habitats. Conversely, less plastic species should be found in only the nonurban habitats. Of course, some aspects of urbanization might in fact alleviate stress on organisms, for example with food supplementation in cities (Rivkin et al. 2019). In this case, a link between plasticity in, for example, resource-acquisition or resource-use traits and persistence would not be expected, and thus could serve as a useful control. While we have laid out an approach to evaluate the pattern of buying time that relies on gathering new data, we note that it might also be feasible to perform such a comparative analysis with data in-hand by using publicly available databases of trait plasticity (e.g., Gunderson and Stillman 2015) and occurrence records (e.g., iNaturalist, GBIF).

\subsubsection{Establishing a Causal Link between Plasticity and Persistence Using Reciprocal Transplant Experiments}

Although the correlation of plasticity with urban success is suggestive of buying time, manipulative experiments are needed to establish a causal link. At either the intra- or inter-specific level, genotypes or species with varying levels of plasticity could be transplanted from the nonurban to urban habitat and monitored with respect to population size. Such an approach might be possible for only a subset of the species that could be assessed for the pattern of buying time, but transplantation would be necessary to directly establish that the plasticity caused population persistence.

\subsubsection{Assessing the Consequences of Buying Time: Does Plasticity Buy Time for Populations to Adapt to Urbanization?}

Finally, once the pattern of buying time was established, and, ideally the causal link between plasticity and persistence as well, these species that were able to persist in urban habitats would be excellent candidates to examine whether plasticity was able to buy time for urban adaptation. Common garden experiments could again be used, but in this case, with both urban and nonurban populations being reared for a complete generation and assessed for divergence in their trait values. 
Here, the expectation would be that species for which plasticity allowed persistence in the novel urban environment also showed adaptive trait divergence from their nonurban ancestors. The magnitude of divergence might also be expected to scale with time since urbanization occurred. That is, more recently urbanized populations would have had less time to adapt.

\subsection{CONCLUSIONS}

Theoretical expectations of plasticity's role in buying time for populations - with or without subsequent evolutionary change-are supported by a number of empirical studies. As a consequence, it seems that there is consensus that buying time via plasticity can occur in natural populations undergoing environmental change. However, there are many unanswered questions in the details of this process (see Box 8.1), and this area harbors most of the controversy surrounding buying time, though we note that there is disagreement among researchers regarding how frequently buying time occurs and for how many systems and taxa. Indeed, the most controversial areas appear to be focused on the subsequent processes following buying time, namely whether plasticity buys time for evolution to occur, and the nature of the plastic response (particularly maladaptive plasticity) and its effects on evolutionary responses.

\section{BOX 8.1 SUGGESTIONS FOR FUTURE RESEARCH}

- What is the relative importance of buying time?

- How much time does plasticity buy for populations? When plasticity fails to buy time, is this simply too small a magnitude of plasticity for the rate of environmental change, maladaptive plasticity, or that plasticity is not associated with population persistence?

- How do we improve tests of buying time? Could a causal link between plasticity and persistence be established with broader tests of the pattern such as via urbanization gradients coupled with manipulative experiments?

- What are the predictors of buying time?

- Is buying time most effective (high population persistence) for developmental selection mechanisms, moderately effective for generalized physiological response mechanisms and least effective for phenotypic switch? Do increasing costs of phenotype for developmental selection mechanisms temper the high probability of being adaptive in the novel environment, such that generalized physiological response mechanisms become most effective?

- What are the costs of phenotypic plasticity in novel environments? Does this vary for different mechanisms of plasticity (i.e., developmental selection, generalized physiological response, phenotypic switch)? 
- What is the distribution effects of fitness effects for plasticity in novel environments? Is plasticity more often adaptive or maladaptive?

- Is buying time more common with directional shifts in the environment versus exposure to completely novel environments?

- What are the consequences of buying time?

- Does plasticity rescue populations outright, i.e., does plasticity buy 'infinite' time? Does plasticity buy time for adaptive evolution to occur? Or does buying time via plasticity simply lead to time lags in population extinction? How often does each of these outcomes occur?

- Is buying time via plasticity rescue more gentle than evolutionary rescue which incurs demographic loss due to selection?

- Is buying time via plasticity effective across a broader range of taxa than evolutionary rescue as it is released from constraints on rapid generation time and the presence of heritable variation?

- Can purely buying time be separated from the evolutionary response after a single generation?

\section{ACKNOWLEDGMENTS}

We thank David Pfennig and two anonymous reviewers for helpful comments on a previous version of this manuscript. SED and RAM were supported by the Oglebay Fund Trust. SED was also supported by an NSF CAREER award (DEB-1845126).

\section{REFERENCES}

Alberdi, A., O. Aizpurua, K. Bohmann, M. L. Zepeda-Mendoza, and M. T. P. Gilbert. 2016. Do vertebrate gut metagenomes confer rapid ecological adaptation? Trends Ecol. Evol. 31:689-699.

Ancel, L. W. 2000. Undermining the Baldwin expediting effect: Does phenotypic plasticity accelerate evolution? Theor. Popul. Biol. 58:307-319.

Ashander, J., L.-M. Chevin, and M. L. Baskett. 2016. Predicting evolutionary rescue via evolving plasticity in stochastic environments. Proc. R. Soc. B 283:20161690.

Aubret, F. and R. Shine. 2009. Genetic assimilation and the postcolonization erosion of phenotypic plasticity in island tiger snakes. Curr. Biol. 19:1932-1936.

Badyaev, A. V. 2009. Evolutionary significance of phenotypic accommodation in novel environments: An empirical test of the Baldwin effect. Philos. Trans. R. Soc. B 364:1125-1141.

Baker, H. G. 1965. Characteristics and modes of origin of weeds, pp. 147-172. In G. L. Stebbins and H. G. Baker, eds., The Genetics of Colonizing Species. Academic Press Inc., New York.

Baldwin, J. M. 1896. A new factor in evolution. Am. Nat. 30:441-451.

Bonamour, S., L.-M. Chevin, A. Charmantier, and C. Teplitsky. 2019. Phenotypic plasticity in response to climate change: The importance of cue variation. Philos. Trans. R. Soc. B 374:20180178. 
Bonduriansky, R. 2021. Plasticity across generations. In D. W. Pfennig, ed., Phenotypic Plasticity and Evolution: Causes, Consequences, Controversies. CRC Press, Boca Raton, FL.

Bradshaw, A. D. 1965. Evolutionary significance of phenotypic plasticity in plants, pp. 115155. In E. W. Caspari and J. M. Thoday, eds., Advances in Genetics. Academic Press, Cambridge, MA.

Campbell-Staton, S. C., K. M. Winchell, N. C. Rochette, J. Fredette, I. Maayan, R. M. Schweizer, and J. Catchen. 2020. Parallel selection on thermal physiology facilitates repeated adaptation of city lizards to urban heat islands. Nat. Ecol. Evol. 4:652-658.

Chevin, L.-M. and A. A. Hoffmann. 2017. Evolution of phenotypic plasticity in extreme environments. Philos. Trans. R. Soc. B 372:20160138.

Chevin, L.-M. and R. Lande. 2011. Adaptation to marginal habitats by evolution of increased phenotypic plasticity. J. Evol. Biol. 24:1462-1476.

Chevin, L.-M., R. Lande, and G. M. Mace. 2010. Adaptation, plasticity, and extinction in a changing environment: Towards a predictive theory. PLoS Biol. 8:e1000357.

Chown, S. L. and S. Nicolson. 2004. Insect Physiological Ecology: Mechanisms and Patterns. Oxford University Press, Oxford.

Chown, S. L., S. Slabber, M. A. McGeoch, C. Janion, and H. P. Leinaas. 2007. Phenotypic plasticity mediates climate change responses among invasive and indigenous arthropods. Proc. R. Soc. B 274:2531-2537.

Corl, A., K. Bi, C. Luke, A. S. Challa, A. J. Stern, B. Sinervo, and R. Nielsen. 2018. The genetic basis of adaptation following plastic changes in coloration in a novel environment. Curr. Biol. 28:2970-2977.

Costa, J. T. 2021. There is hardly any question in biology of more importance-Charles Darwin and the nature of variation. In D. W. Pfennig, ed., Phenotypic Plasticity and Evolution: Causes, Consequences, Controversies. CRC Press, Boca Raton, FL.

Crispo, E. 2007. The Baldwin effect and genetic assimilation: Revisiting two mechanisms of evolutionary change mediated by phenotypic plasticity. Evolution 61:2469-2479.

Crispo, E., J. D. DiBattista, C. Correa, X. Thibert-Plante, A. E. McKellar, A. K. Schwartz, D. Berner, L. F. D. León, and A. P. Hendry. 2010. The evolution of phenotypic plasticity in response to anthropogenic disturbance. Evol. Ecol. Res. 12:47-66.

Davidson, A. M., M. Jennions, and A. B. Nicotra. 2011. Do invasive species show higher phenotypic plasticity than native species and, if so, is it adaptive? A meta-analysis. Ecol. Lett. 14:419-431.

Diamond, S. E. and R. A. Martin. 2016. The interplay between plasticity and evolution in response to human-induced environmental change. F1000Research 5:2835.

Diamond, S. E. and R. A. Martin. 2020. Evolution is a double-edged sword, not a silver bullet, to confront climate change. Ann. N. Y. Acad. Sci 1469:38-51.

Diamond, S. E., L. D. Chick, A. Perez, S. A. Strickler, and R. A. Martin. 2018. Evolution of thermal tolerance and its fitness consequences: Parallel and non-parallel responses to urban heat islands across three cities. Proc. R. Soc. B 285:20180036.

Donelson, J. M., S. Salinas, P. L. Munday, and L. N. Shama. 2018. Transgenerational plasticity and climate change experiments: Where do we go from here? Glob. Change Biol. 24:13-34.

Donohue, K., E. H. Pyle, D. Messiqua, M. S. Heschel, and J. Schmitt. 2001. Adaptive divergence in plasticity in natural populations of Impatiens capensis and its consequences for performance in novel habitats. Evolution 55:692-702.

Ducatez, S., D. Sol, F. Sayol, and L. Lefebvre. 2020. Behavioural plasticity is associated with reduced extinction risk in birds. Nat. Ecol. Evol. 4(6):1-6.

Fox, R. J., J. M. Donelson, C. Schunter, T. Ravasi, and J. D. Gaitán-Espitia. 2019. Beyond buying time: The role of plasticity in phenotypic adaptation to rapid environmental change. Philos. Trans. R. Soc. B 374:20180174. 
Frank, S. A. 2011. Natural selection. II. Developmental variability and evolutionary rate. J. Evol. Biol. 24:2310-2320.

Funk, J. L. 2008. Differences in plasticity between invasive and native plants from a low resource environment. J. Ecol. 96:1162-1173.

Futuyma, D. J. 2021. How does phenotypic plasticity fit into evolutionary theory? In D. W. Pfennig, ed., Phenotypic Plasticity and Evolution: Causes, Consequences, Controversies. CRC Press, Boca Raton, FL.

Gause, G. 1942. The relation of adaptability to adaptation. Q. Rev. Biol. 17:99-114.

Ghalambor, C. K., J. K. McKAY, S. P. Carroll, and D. N. Reznick. 2007. Adaptive versus non-adaptive phenotypic plasticity and the potential for contemporary adaptation in new environments. Funct. Ecol. 21:394-407.

Gomez-Mestre, I., and D. R. Buchholz. 2006. Developmental plasticity mirrors differences among taxa in spadefoot toads linking plasticity and diversity. PNAS 103:19021-19026.

Grether, G. F. 2005. Environmental change, phenotypic plasticity, and genetic compensation. Am. Nat. 166:E115-E123.

Gunderson, A. R. and J. H. Stillman. 2015. Plasticity in thermal tolerance has limited potential to buffer ectotherms from global warming. Proc. R. Soc. B 282:20150401.

Hahn, M. A., M. van Kleunen, and H. Müller-Schärer. 2012. Increased phenotypic plasticity to climate may have boosted the invasion success of polyploid Centaurea stoebe. PLoS ONE 7:e50284.

Hinton, G. E. and S. J. Nowlan. 1987. How learning can guide evolution. Complex Sys. $1: 495-502$.

Hua, J., D. K. Jones, B. M. Mattes, R. D. Cothran, R. A. Relyea, and J. T. Hoverman. 2015. The contribution of phenotypic plasticity to the evolution of insecticide tolerance in amphibian populations. Evol. Appl. 8:586-596.

Kolbe, J. J. and J. B. Losos. 2005. Hind-Limb length plasticity in Anolis carolinensis. J. Herpetol. 39:674-678.

Kulkarni, S. S., R. J. Denver, I. Gomez-Mestre, and D. R. Buchholz. 2017. Genetic accommodation via modified endocrine signalling explains phenotypic divergence among spadefoot toad species. Nat. Commun. 8:993.

Lande, R. 2009. Adaptation to an extraordinary environment by evolution of phenotypic plasticity and genetic assimilation. J. Evol. Biol. 22:1435-1446.

Lande, R. 2014. Evolution of phenotypic plasticity and environmental tolerance of a labile quantitative character in a fluctuating environment. J. Evol. Biol. 27:866-875.

Lande, R. 2015. Evolution of phenotypic plasticity in colonizing species. Mol. Ecol. 24:2038-2045.

Levin, D. A. 1983. Polyploidy and novelty in flowering plants. Am. Nat. 122:1-25.

Levis, N. A. and D. W. Pfennig. 2019. How stabilizing selection and nongenetic inheritance combine to shape the evolution of phenotypic plasticity. J. Evol. Biol. 32:706-716.

Lister, A. M. 2021. Phenotypic plasticity in the fossil record. In D. W. Pfennig, ed., Phenotypic Plasticity and Evolution: Causes, Consequences, Controversies. CRC Press, Boca Raton, FL.

Losos, J. B., D. A. Creer, D. Glossip, R. Goellner, A. Hampton, G. Roberts, N. Haskell, P. Taylor, and J. Ettling. 2000. Evolutionary implications of phenotypic plasticity in the hindlimb of the lizard Anolis sagrei. Evolution 54:301-305.

Markov, A. V. and S. B. Ivnitsky. 2016. Evolutionary role of phenotypic plasticity. Moscow Univ. Biol. Sci. Bull. 71:185-192.

Martin, R. A. and D. W. Pfennig. 2010. Maternal investment influences expression of resource polymorphism in amphibians: Implications for the evolution of novel resource-use phenotypes. PLoS ONE 5:e9117.

Matesanz, S., T. Horgan-Kobelski, and S. E. Sultan. 2012. Phenotypic plasticity and population differentiation in an ongoing species invasion. PLOS ONE 7:e44955. 
Merilä, J. and A. P. Hendry. 2014. Climate change, adaptation, and phenotypic plasticity: The problem and the evidence. Evol. Appl. 7:1-14.

Morgan, C. L. 1896. On modification and variation. Science 4:733-740.

Morris, M. R. J. 2014. Plasticity-mediated persistence in new and changing environments. Int. J. Evol. Biol. 2014:416497.

Muñoz, M. M. and J. B. Losos. 2017. Thermoregulatory behavior simultaneously promotes and forestalls evolution in a tropical lizard. Am. Nat. 191:E15-E26.

Muschick, M., M. Barluenga, W. Salzburger, and A. Meyer. 2011. Adaptive phenotypic plasticity in the Midas cichlid fish pharyngeal jaw and its relevance in adaptive radiation. BMC Evol. Biol. 11:116.

Neel, L. K., J. D. Curlis, C. T. Kinsey, C. L. Cox, and L. D. McBrayer. 2020. Acclimatization in the physiological performance of an introduced ectotherm. J. Exp. Biol. 223:jeb201517.

Nunney, L. 2016. Adapting to a changing environment: Modeling the interaction of directional selection and plasticity. J. Hered. 107:15-24.

Nyamukondiwa, C., E. Kleynhans, and J. S. Terblanche. 2010. Phenotypic plasticity of thermal tolerance contributes to the invasion potential of Mediterranean fruit flies (Ceratitis capitata). Ecol. Entomol. 35:565-575.

Osborn, H. F. 1896. A mode of evolution requiring neither natural selection nor the inheritance of acquired characters. Trans. N. Y. Acad. Sci. 15:141-142.

Paenke, I., B. Sendhoff, and T. J. Kawecki. 2007. Influence of plasticity and learning on evolution under directional selection. Am. Nat. 170:E47-E58.

Pfennig, D. W. 2021. Key questions about phenotypic plasticity. In D. W. Pfennig, ed., Phenotypic Plasticity and Evolution: Causes, Consequences, Controversies. CRC Press, Boca Raton, FL.

Pfennig, D. W. and R. A. Martin. 2009. A maternal effect mediates rapid population divergence and character displacement in spadefoot toads. Evolution 63:898-909.

Pfennig, D. W. and M. McGee. 2010. Resource polyphenism increases species richness: A test of the hypothesis. Philos. Trans. R. Soc. B 365:577-591.

Pfennig, D. W. and P. J. Murphy. 2000. Character displacement in polyphenic tadpoles. Evolution 54:1738-1749.

Pfennig, D. W., and P. J. Murphy. 2002. How fluctuating competition and phenotypic plasticity mediate species divergence. Evolution 56:1217-1228.

Pfennig, D. W., and K. S. Pfennig. 2012. Development and evolution of character displacement. Ann. N. Y. Acad. Sci. 1256:89-107.

Pfennig, D. W., A. M. Rice, and R. A. Martin. 2006. Ecological opportunity and phenotypic plasticity interact to promote character displacement and species coexistence. Ecology 87:769-779.

Pfennig, D. W., A. M. Rice, and R. A. Martin. 2007. Field and experimental evidence for competition's role in phenotypic divergence. Evolution 61:257-271.

Pigliucci, M. 2001. Phenotypic Plasticity: Beyond Nature and Nurture. JHU Press, Baltimore, MD.

Price, T. D., A. Qvarnström, and D. E. Irwin. 2003. The role of phenotypic plasticity in driving genetic evolution. Proc. R. Soc. B 270:1433-1440.

Radchuk, V., T. Reed, C. Teplitsky, M. van de Pol, A. Charmantier, C. Hassall, P. Adamík, F. Adriaensen, M. P. Ahola, P. Arcese, J. M. Avilés, J. Balbontin, K. S. Berg, A. Borras, S. Burthe, J. Clobert, N. Dehnhard, F. de Lope, A. A. Dhondt, N. J. Dingemanse, H. Doi, T. Eeva, J. Fickel, I. Filella, F. Fossøy, A. E. Goodenough, S. J. G. Hall, B. Hansson, M. Harris, D. Hasselquist, T. Hickler, J. Joshi, H. Kharouba, J. G. Martínez, J.-B. Mihoub, J. A. Mills, M. Molina-Morales, A. Moksnes, A. Ozgul, D. Parejo, P. Pilard, M. Poisbleau, F. Rousset, M.-O. Rödel, D. Scott, J. C. Senar, C. Stefanescu, B. G. Stokke, T. Kusano, M. Tarka, C. E. Tarwater, K. Thonicke, J. Thorley, A. Wilting, P. Tryjanowski, J. Merilä, 
B. C. Sheldon, A. P. Møller, E. Matthysen, F. Janzen, F. S. Dobson, M. E. Visser, S. R. Beissinger, A. Courtiol, and S. Kramer-Schadt. 2019. Adaptive responses of animals to climate change are most likely insufficient. Nat. Commun. 10:1-14.

Reed, T. E., R. S. Waples, D. E. Schindler, J. J. Hard, and M. T. Kinnison. 2010. Phenotypic plasticity and population viability: The importance of environmental predictability. Proc. R. Soc. B 277:3391-3400.

Reznick, D. N., J. Losos, and J. Travis. 2019. From low to high gear: There has been a paradigm shift in our understanding of evolution. Ecol. Lett. 22:233-244.

Richards, C. L., O. Bossdorf, N. Z. Muth, J. Gurevitch, and M. Pigliucci. 2006. Jack of all trades, master of some? On the role of phenotypic plasticity in plant invasions. Ecol. Lett. 9:981-993.

Rivkin, L. R., J. S. Santangelo, M. Alberti, M. F. J. Aronson, C. W. de Keyzer, S. E. Diamond, M.-J. Fortin, L. J. Frazee, A. J. Gorton, A. P. Hendry, Y. Liu, J. B. Losos, J. S. MacIvor, R. A. Martin, M. J. McDonnell, L. S. Miles, J. Munshi-South, R. W. Ness, A. E. M. Newman, M. R. Stothart, P. Theodorou, K. A. Thompson, B. C. Verrelli, A. Whitehead, K. M. Winchell, and M. T. J. Johnson. 2019. A roadmap for urban evolutionary ecology. Evol. Appl. 12:384-398.

Robinson, B. W. and R. Dukas. 1999. The influence of phenotypic modifications on evolution: The Baldwin effect and modern perspectives. Oikos 85:582-589.

Scheiner, S. M. 1993. Genetics and evolution of phenotypic plasticity. Annu. Rev. Ecol. Evol. Syst. 24:35-68.

Scheiner, S. M. 2014. The Baldwin effect: Neglected and misunderstood. Am. Nat. 184:2-3.

Scheiner, S. M., M. Barfield, and R. D. Holt. 2017. The genetics of phenotypic plasticity. XV. Genetic assimilation, the Baldwin effect, and evolutionary rescue. Ecol. Evol. 7:8788-8803.

Scheiner, S. M., M. Barfield, and R. D. Holt. 2020. The genetics of phenotypic plasticity. XVII. Response to climate change. Evol. Appl. 13:388-399.

Schlichting, C. D. and M. A. Wund. 2014. Phenotypic plasticity and epigenetic marking: An assessment of evidence for genetic accommodation. Evolution 68:656-672.

Schmalhausen, I. I. 1949. Factors of Evolution: The Theory of Stabilizing Selection. Blakiston, Philadelphia, PA.

Simpson, G. G. 1953. The Baldwin effect. Evolution 7:110-117.

Sinervo, B., F. Méndez-de-la-Cruz, D. B. Miles, B. Heulin, E. Bastiaans, M. V.-S. Cruz, R. Lara-Resendiz, N. Martínez-Méndez, M. L. Calderón-Espinosa, R. N. Meza-Lázaro, H. Gadsden, L. J. Avila, M. Morando, I. J. D. la Riva, P. V. Sepulveda, C. F. D. Rocha, N. Ibargüengoytía, C. A. Puntriano, M. Massot, V. Lepetz, T. A. Oksanen, D. G. Chapple, A. M. Bauer, W. R. Branch, J. Clobert, and J. W. Sites. 2010. Erosion of lizard diversity by climate change and altered thermal niches. Science 328:894-899.

Snell-Rood, E. C., M. E. Kobiela, K. L. Sikkink, and A. M. Shephard. 2018. Mechanisms of plastic rescue in novel environments. Annu. Rev. Ecol. Evol. Syst. 49:331-354.

Sol, D., S. Timmermans, and L. Lefebvre. 2002. Behavioural flexibility and invasion success in birds. Anim. Behav. 63:495-502.

Sol, D., R. P. Duncan, T. M. Blackburn, P. Cassey, and L. Lefebvre. 2005. Big brains, enhanced cognition, and response of birds to novel environments. PNAS 102:5460-5465.

Sørensen, J. G., T. N. Kristensen, and J. Overgaard. 2016. Evolutionary and ecological patterns of thermal acclimation capacity in Drosophila: Is it important for keeping up with climate change? Curr. Opin. Insect Sci. 17:98-104.

Telemeco, R. S., B. Fletcher, O. Levy, A. Riley, Y. Rodriguez-Sanchez, C. Smith, C. Teague, A. Waters, M. J. Angilletta, and L. B. Buckley. 2017. Lizards fail to plastically adjust nesting behavior or thermal tolerance as needed to buffer populations from climate warming. Glob. Change Biol. 23:1075-1084. 
Thibert-Plante, X. and A. Hendry. 2011. The consequences of phenotypic plasticity for ecological speciation. J. Evol. Biol. 24:326-342.

Trussell, G. C. and L. D. Smith. 2000. Induced defenses in response to an invading crab predator: An explanation of historical and geographic phenotypic change. PNAS 97:2123-2127.

Van de Peer, Y., E. Mizrachi, and K. Marchal. 2017. The evolutionary significance of polyploidy. Nat. Rev. Genet. 18:411-424.

Via, S. and R. Lande. 1985. Genotype-environment interaction and the evolution of phenotypic plasticity. Evolution 39:505-522.

Volis, S., D. Ormanbekova, and K. Yermekbayev. 2015. Role of phenotypic plasticity and population differentiation in adaptation to novel environmental conditions. Ecol. Evol. 5:3818-3829.

Volis, S., D. Ormanbekova, K. Yermekbayev, M. Song, and I. Shulgina. 2014. Introduction beyond a species range: A relationship between population origin, adaptive potential and plant performance. Heredity 113:268-276.

Waddington, C. H. 1961. Genetic assimilation, pp. 257-293. In E. W. C. and J. M. Thoday, eds., Advances in Genetics. Academic Press, Cambridge, MA.

Wcislo, W. T. 1989. Behavioral environments and evolutionary change. Annu. Rev. Ecol. Evol. Syst. 20:137-169.

Wei, N., R. Cronn, A. Liston, and T.-L. Ashman. 2019. Functional trait divergence and trait plasticity confer polyploid advantage in heterogeneous environments. New Phytol. 221:2286-2297.

West-Eberhard, M. J. 1989. Phenotypic plasticity and the origins of diversity. Annu. Rev. Ecol. Evol. Syst. 20:249-278.

West-Eberhard, M. J. 2003. Developmental Plasticity and Evolution. Oxford University Press, Oxford.

Wund, M. A. 2012. Assessing the impacts of phenotypic plasticity on evolution. Integr. Comp. Biol. 52:5-15.

Wund, M. A., J. A. Baker, B. Clancy, J. L. Golub, and S. A. Foster. 2008. A test of the "flexible stem" model of evolution: Ancestral plasticity, genetic accommodation, and morphological divergence in the threespine stickleback radiation. Am. Nat. 172:449-462.

Yeh, P. J. and T. D. Price. 2004. Adaptive phenotypic plasticity and the successful colonization of a novel environment. Am. Nat. 164:531-542. 